\title{
Association of mitochondrial DNA copy number with self-rated health status
}

This article was published in the following Dove Press journal: The Application of Clinical Genetics

\author{
Paul Y Takahashi' \\ Gregory D Jenkins ${ }^{2}$ \\ Benjamin P Welkie ${ }^{2}$ \\ Shannon K McDonnell ${ }^{2}$ \\ Jared M Evans ${ }^{2}$ \\ James R Cerhan² \\ Janet E Olson ${ }^{2}$ \\ Stephen N Thibodeau ${ }^{3}$ \\ Mine S Cicek ${ }^{3}$ \\ Euijung Ryu ${ }^{2}$ \\ 'Division of Primary Care Internal \\ Medicine, ${ }^{2}$ Department of Health \\ Sciences Research, ${ }^{3}$ Department of \\ Laboratory Medicine and Pathology, \\ Mayo Clinic, Rochester, MN, USA
}

Correspondence: Paul Y Takahashi Division of Primary Care Internal Medicine, Mayo Clinic, 200 First Street SW, Rochester, MN 55905, USA

$\mathrm{Tel}+\mathrm{I} 50728425$ I I

Fax + I 507266278 I

Email takahashi.paul@mayo.edu
Purpose: In aging adults, mitochondrial dysfunction may be an important contributor. We evaluated the association between mitochondrial DNA (mtDNA) copy number, which is a biomarker for mitochondrial function, and self-rated health status.

Patients and methods: We conducted a cross-sectional study of patients enrolled within the Mayo Clinic Biobank. We utilized the questionnaire and sequence data from 944 patients. We examined the association between mtDNA copy number and self-rated health status with 3 collapsed categories for the latter variable (excellent/very good, good, and fair/poor). For analysis, we used proportional odds models after log-transforming mtDNA copy number, and we adjusted for age and sex.

Results: We found the median age at enrollment was 61 years (25th-75th percentile: $51-71)$, and $64 \%$ reported excellent or very good health, $31 \%$ reported good health, and $6 \%$ reported fair/poor health. Overall, the median mtDNA copy number was 88.9 (25th-75th percentile: 77.6-101.1). Higher mtDNA copy number was found for subjects reporting better self-rated health status after adjusting for age, sex, and comorbidity burden ( $\mathrm{OR}=2.3$ [95\% CI: 1.2-4.5] for having better self-rated health for a one-unit increase in log-transformed mtDNA copy number). Conclusion: We found that a higher mtDNA copy number is associated with better self-rated health status after adjustment for age, sex, and comorbidity burden. The current study implies that mtDNA copy number may serve as a biomarker for self-reported health. Further studies, potentially including cohort studies, may be required.

Keywords: mitochondrial DNA copy number, self-rated health, personalized medicine

\section{Introduction}

Patient-reported outcomes and self-rated health are important predictors for many adverse health outcomes including mortality. ${ }^{1}$ Participants with worse self-rated physical health have had a higher risk of hospitalization. ${ }^{2}$ Poor self-rated health is also associated with all-cause and cardiovascular-related mortality. ${ }^{3}$ Despite the association between self-rated health and adverse health outcomes, self-rated general health can be viewed to be subjective. The relationship between self-rated outcomes and biomarkers is less clear and an area for potential investigation. In some initial studies, there appeared to be a graded relationship between poor self-rated health and higher white blood cell count, lower hemoglobin count, and higher creatinine levels. ${ }^{4}$ Poor self-rated health has also been associated with elevated levels of interleukin 6 and C-reactive protein, immune-related biomarkers, in healthy older adults. ${ }^{5}$ Dantzer and Walker suggested that inflammation may serve as a biological mechanism for an exacerbation of sickness in physically ill people by regulating symptoms of sickness 
(e.g., pain, fatigue, and decreased social and physical activity), which leads to depression and subsequently affects the quality of life including self-rated health. ${ }^{6}$ Thus, self-rated health may reflect biological changes.

The role of the mitochondria has been receiving increasing attention in various health-related research supported by substantial evidence of a causative link between mitochondrial dysfunction and aging and health outcomes. ${ }^{7}$ Additionally, it is suggested that the link between inflammation and health conditions may be modulated by mitochondrial dysfunction..$^{8-11}$ Since mitochondrial function is regulated by both the nuclear and mitochondrial genomes, it has been proposed that variation in mitochondrial DNA (mtDNA), an understudied human genome compared to the nuclear genome, may also play an important role in these health conditions. ${ }^{12}$ Additionally, mtDNA mutations are accumulated over a lifetime with several risk factors associated with adverse outcomes, such as smoking exposure, leading to a faster accumulation rate. ${ }^{13}$ mtDNA copy number has been suggested to be a link between risk factors and health outcomes. For example, the association between smoking and lung cancer may be explained by changes in mtDNA copy numbers, due to increased oxidative stress and increased somatic mtDNA mutations caused by smoking, which leads to mitochondrial dysfunction. ${ }^{14}$

There are few studies that examine the correlation between mtDNA contents and quality of life (e.g., self-rated overall health). To the best of our knowledge, there is only one study regarding an association between mtDNA copy number and self-rated general health which showed that higher mtDNA copy number in blood cells is associated with better overall health among elderly individuals. ${ }^{14}$ That study showed no sex difference in terms of mtDNA copy number. This finding is in conflict with another study reporting that mtDNA copy number was significantly higher in women. ${ }^{15}$ In addition, the age relationship has been inconclusive. The authors of the former study reported that a decline in mtDNA was not observed until the age of 48 years. ${ }^{14}$ Other researchers have reported that mtDNA copy number slightly increased until the fifth decade of life and declined in older subjects. ${ }^{15}$ Thus, there appears to be some disagreement regarding the relationship between mtDNA copy number and basic demographics as well as self-rated health.

In the current study, we aimed to assess whether mtDNA copy number was associated with self-rated overall health, independent of basic demographic characteristics. Because mitochondrial dysfunction is observed in numerous chronic diseases and linked with other non-specific symptoms (e.g., excessive fatigue) that are common in nearly all chronic diseases, ${ }^{16}$ we hypothesized that mtDNA copy number, a biomarker for mitochondrial dysfunction, would be associated with self-rated health status.

\section{Patients and methods Study design and participants}

This was a cross-sectional study of patients enrolled in the Mayo Clinic Biobank (MCB) for whom whole genome sequencing (WGS) data were available along with selfreported questionnaires completed at enrollment which included self-rated general health. Participants were patients receiving continuous medical care at Mayo Clinic. Among those enrolled as of March 30, 2018, $1000 \mathrm{MCB}$ participants were selected based on sex-stratified random sampling approach (i.e., 500/500 males/females) for a WGS experiment. The governance and specifics of the biobank have been noted previously. ${ }^{17}$ Participants were excluded if they withdrew consent from the biobank. Patients provided written consent for use of their biological samples, questionnaire data, and electronic medical record data as part of enrollment within the biobank..$^{18}$ The study was reviewed and approved by the Mayo Clinic Institutional Review Board and Mayo Clinic Biobank Access committee.

\section{WGS experiment and bioinformatics}

Extracted DNA from peripheral blood samples was sequenced on the Illumina HiSeq X10 with 150 bp paired end reads and a target average read depth of $30 \times$. Comprehensive bioinformatics analysis was performed for read alignment to the hg38 genome, variant calling, and annotation. Extensive sample quality controls were performed, including sample contamination and relatedness check. Estimates of sample contamination were obtained using the VerifyBamID sequence-only method with the 1000 genomes array reference. ${ }^{19}$ Samples with high contamination score (FREEMIX $\geq 0.04$ ) were excluded. ${ }^{34}$ The relatedness of each pair of subjects was evaluated by estimating the kinship coefficient using the KING-robust procedure, ${ }^{20}$ as implemented in the R package SNPRelate. ${ }^{21}$ Among the pairs of first- or second-degree relatives, those with lower call rates were excluded. To identify reads associated with mtDNA, the revised Cambridge Reference Sequence ${ }^{22}$ was used for alignment, and basic quality filtering was applied before single nucleotide variant calling.

\section{Measurement of mtDNA contents}

Once both autosomal and mtDNA sequence reads were aligned, average sequencing coverage was compared to 
quantify mtDNA copy number, which was defined as the relative abundance of mtDNA compared to autosomal DNA (i.e., 2 times mtDNA average coverage, divided by autosomal DNA average coverage). ${ }^{23}$ In addition, mitochondrial haplogroups were estimated using HaploGrep. ${ }^{24}$ Major European haplogroups were collapsed into 5 groups (HV [including HV, $\mathrm{H}$, and V], J, T, K, U-K [U haplogroups excluding K subclade], and other European haplogroups). All non-European haplogroups were combined (i.e., non-European haplogroups).

\section{Correlates}

The primary variable of interest was self-rated health as noted on the initial questionnaire upon entry into the biobank. The questionnaire listed 5 categories of self-rated health as poor, fair, good, very good, and excellent. We reported 3 categories of self-rated health as poor/fair, good, and very good/ excellent. The other variables included age, sex, education attainment, alcohol consumption, smoking status, body mass index (BMI), and number of comorbidities. Age, sex, BMI, and the number of comorbidities were obtained from patients' electronic medical record. The number of comorbidities at the time of sample collection was calculated by counting 20 selected chronic conditions recommended by the US Department of Health and Human Services. ${ }^{25}$ Other variables were collected from self-reported questionnaires: education attainment (high school or less, some college, college graduate, or graduate/professional degree), alcohol use (never, once a month, 2-4 times a month, 2-5 times a week, $>6$ times a week), and tobacco use (ever vs never).

\section{Statistical analysis}

Basic demographic characteristics and mitochondrial content information (mtDNA copy number and mitochondrial haplogroups) were presented using summary statistics, for the overall cohort as well as ones stratified by perceived general health (poor/fair, good, very good/excellent). Univariately, each variable (including self-rated general health and mtDNA copy number) was first tested using proportional odds models for association with mtDNA copy number. The primary analysis was to test association between mtDNA copy number (log-transformed) and self-rated health, adjusting for age (in quadratic spline) and sex. Additional analysis was performed after adding other pertinent variables such as the number of comorbidities and BMI. As a secondary analysis, multivariable analysis was also performed to determine whether self-rated general health was independently associated with mtDNA copy number, adjusting for all the variables that were univariately associated $(P<0.05)$ with self-rated health.

\section{Results \\ Sample description}

A total of 944 participants had both WGS and questionnaire data. Of those, 601 (64\%) patients had excellent or very good self-rated health compared to 287 (30\%) with good health and $54(5.7 \%)$ with fair/poor health. The median age of the overall sample was 60.5 years $(25 \mathrm{th}-75$ th percentiles: $51.0-71.0)$ and $470(50 \%)$ were female. In the study cohorts, $82 \%$ had some college or higher education. For mtDNA characteristics, the median mtDNA copy number was 88.9 (25th-75th percentiles: 77.6-101.1). The most common mitochondrial haplogroup for the overall cohort was HV with 467 (49.4\%) patients, while $98 \%$ of them belonged to European haplogroups (Table 1).

\section{Association between subject characteristics and self-rated general health}

Univariately, younger age, female sex, lower BMI, higher education level, non-smokers, alcohol consumption, and lower comorbidity burden were associated with better selfrated health (Table 1 and Figure 1). Overall, higher mtDNA copy number was associated with better self-rated health, while mitochondrial haplogroups were not associated (Table 1). Women and younger participants overall tended to have higher mtDNA copy numbers, while the number in women remained relatively stable until they reached their 60s (Figure 2). Adjusting for age (in a quadratic form) and sex, higher mtDNA copy number was observed with subjects reporting better self-rated health status (Table 2). The association remained significant, after adjusting for age, sex, and comorbidity burden (OR $=2.3$ [95\% CI: 1.2-4.5] for having better self-rated health for a one-unit increase in log-transformed mtDNA copy number). A similar association was observed in the model after adjusting for BMI (OR $=2.01$ [95\% CI: 1.00-4.09]). After adjusting for all the variables univariately, the association was no longer significant ( $P=0.14$ ), potentially due to the lack of statistical power and the interrelationship among some of the demographic variables (e.g., alcohol consumption), mtDNA copy number, and self-rated health.

\section{Discussion}

In this study of 956 participants, we found that patients with higher mtDNA copy number in peripheral blood had better self-rated health independent of age. We also found that older patients had lower mtDNA copy numbers. Lastly, we found 
Table I Basic characteristics and mitochondrial DNA information of study subjects, stratified by perceived general health

\begin{tabular}{|c|c|c|c|c|c|}
\hline & \multirow{2}{*}{$\begin{array}{l}\text { Overall } \\
\text { cohort } \\
(n=944)\end{array}$} & \multicolumn{4}{|c|}{ Perceived general health } \\
\hline & & $\begin{array}{l}\text { Excellent/ } \\
\text { very good } \\
(n=60 I)\end{array}$ & $\begin{array}{l}\text { Good } \\
(n=287)\end{array}$ & $\begin{array}{l}\text { Fair/poor } \\
(n=54)\end{array}$ & $P$-value* \\
\hline$\overline{\text { Age, }}$ & 60.5 & 57.0 & 64 & 66.5 & $<0.001$ \\
\hline Median (25th-75th percentile) & $(5 I .0,71.0)$ & $(48.0,67.0)$ & $(53.0,72.0)$ & $(55.3,78.0)$ & \\
\hline Female sex, $\mathrm{N}(\%)$ & $470(49.8)$ & $326(54.2)$ & $|2|(42.2)$ & $22(40.7)$ & $<0.001$ \\
\hline BMI & 28.2 & 27.1 & 30.2 & 30.3 & $<0.001$ \\
\hline Median (25th-75th percentile) & $(24.9,32.4)$ & $(24.3,31.0)$ & $(26.9,34.5)$ & $(26.4,35.4)$ & \\
\hline Education, $\mathrm{N}(\%)$ & & & & & $<0.001$ \\
\hline High school or less & $167(18.0)$ & $79(13.3)$ & $70(25.0)$ & $18(36.0)$ & \\
\hline Some college & $326(35.2)$ & $201(33.8)$ & $110(39.3)$ & $15(30.0)$ & \\
\hline College & $257(27.7)$ & $182(30.6)$ & $61(21.8)$ & $13(26.0)$ & \\
\hline Graduate/professional & $177(19.1)$ & $133(22.4)$ & $39(13.9)$ & $4(8.0)$ & \\
\hline Alcohol consumption, $\mathrm{N}(\%)$ & & & & & $<0.001$ \\
\hline Never & $171(18.1)$ & $86(12.8)$ & $63(22.0)$ & $22(40.7)$ & \\
\hline Once a month or less & $206(21.8)$ & $188(28.0)$ & $71(24.7)$ & $16(29.6)$ & \\
\hline $2-4$ times a month & $245(26.0)$ & $168(25.0)$ & $69(24.0)$ & $8(14.8)$ & \\
\hline 2-5 times a week & $254(26.9)$ & $184(27.4)$ & $63(22.0)$ & $7(13.0)$ & \\
\hline $6+$ a week & $67(7.1)$ & $45(6.7)$ & $21(7.3)$ & $\mathrm{I}(1.9)$ & \\
\hline Smoking ever, N (\%) & $360(38.1)$ & $194(32.6)$ & $|4|(50.2)$ & $25(48.1)$ & $<0.001$ \\
\hline Number of comorbidities & 3 & 3 & 5 & 7 & $<0.001$ \\
\hline Median (25th-75th percentile) & $(2,6)$ & $(I, 4)$ & $(3,7)$ & $(5,9)$ & \\
\hline Mitochondrial haplogroup, N (\%) & & & & & 0.98 \\
\hline $\mathrm{HV}(\mathrm{HV}, \mathrm{H}$, and $\mathrm{V})$ & $467(49.4)$ & $294(48.9)$ & $144(50.2)$ & $28(51.9)$ & \\
\hline J & $90(9.5)$ & $58(9.7)$ & $26(9.1)$ & $5(9.3)$ & \\
\hline $\mathrm{T}$ & $99(10.5)$ & $66(11.0)$ & $30(10.5)$ & $3(5.6)$ & \\
\hline $\mathrm{U}$ (minus $\mathrm{K}$ ) & $146(15.5)$ & $91(15.1)$ & $45(15.7)$ & $10(18.5)$ & \\
\hline $\mathrm{K}$ & $71(7.5)$ & $47(7.8)$ & $20(7.0)$ & $4(7.4)$ & \\
\hline Other European groups & $55(5.9)$ & $36(6.0)$ & $17(5.9)$ & $3(5.6)$ & \\
\hline Non-European groups & $15(1.6)$ & $9(1.5)$ & $5(1.7)$ & I (I.9) & \\
\hline Mitochondrial copy number & 88.9 & 90.7 & 86.1 & 82.9 & $<0.001$ \\
\hline Median (25th-75th percentile) & $(77.6,101.1)$ & $(79.2,103.3)$ & $(75.8,97.4)$ & $(72.4,99.7)$ & \\
\hline
\end{tabular}

Notes: ${ }^{*}$-values are to test association between each variable and perceived general heath univariately, using proportional odds model.

Abbreviation: BMI, body mass index.

that men had lower mtDNA copy numbers than women. These findings are unique and differ from previous studies. These findings should continue to add to our understanding of the relationship of mtDNA copy number to self-rated health as well as the ongoing work on mtDNA and age and gender.

There has been limited previous work in determining the relationship between self-rated health and mtDNA copy number. In one previous study, 1067 combined peripheral blood samples were examined for such an association. ${ }^{14}$ The authors found a positive association between better selfrated health and higher mtDNA copy numbers. ${ }^{14}$ Our study provides further evidence of the relationship between lower self-rated health and lower mtDNA copy number.

In addition to this main finding, we found that older age was associated with lower mtDNA copy number. The relationship between age and mtDNA copy number depended upon the patient's sex. Previous studies, looking at different tissue types, also showed an age-related decline in mtDNA copy number. ${ }^{26}$ In 3 generations of women in 18 different families, there appeared to be a decline in mtDNA copy number with advancing age. ${ }^{27}$ In the Danish study described earlier, after 48 years of age, there appeared to be 5.4 fewer mtDNA copies per decade. ${ }^{14}$ Some studies have shown different variations in mtDNA copy number above the age of 90 years. ${ }^{28}$ The role of sex and mtDNA copy number differs in our study from previous studies. We found that men had a lower copy number than women. In the Danish study, there was no difference in peripheral blood mtDNA copy number between men and women. ${ }^{14}$ To account for the association we found, we adjusted the final model for both age and sex.

Biologically and clinically, there is importance to this work. Biologically, the changes in the number of the mitochondria DNA with age may reflect an increase in both inflammation and oxidative stress. ${ }^{29}$ In a study of 146 healthy men and women, a reduction in mtDNA number in muscle was associated with an increase in the oxidative DNA lesion, 8-oxo-deoxyguanosine. ${ }^{30}$ 

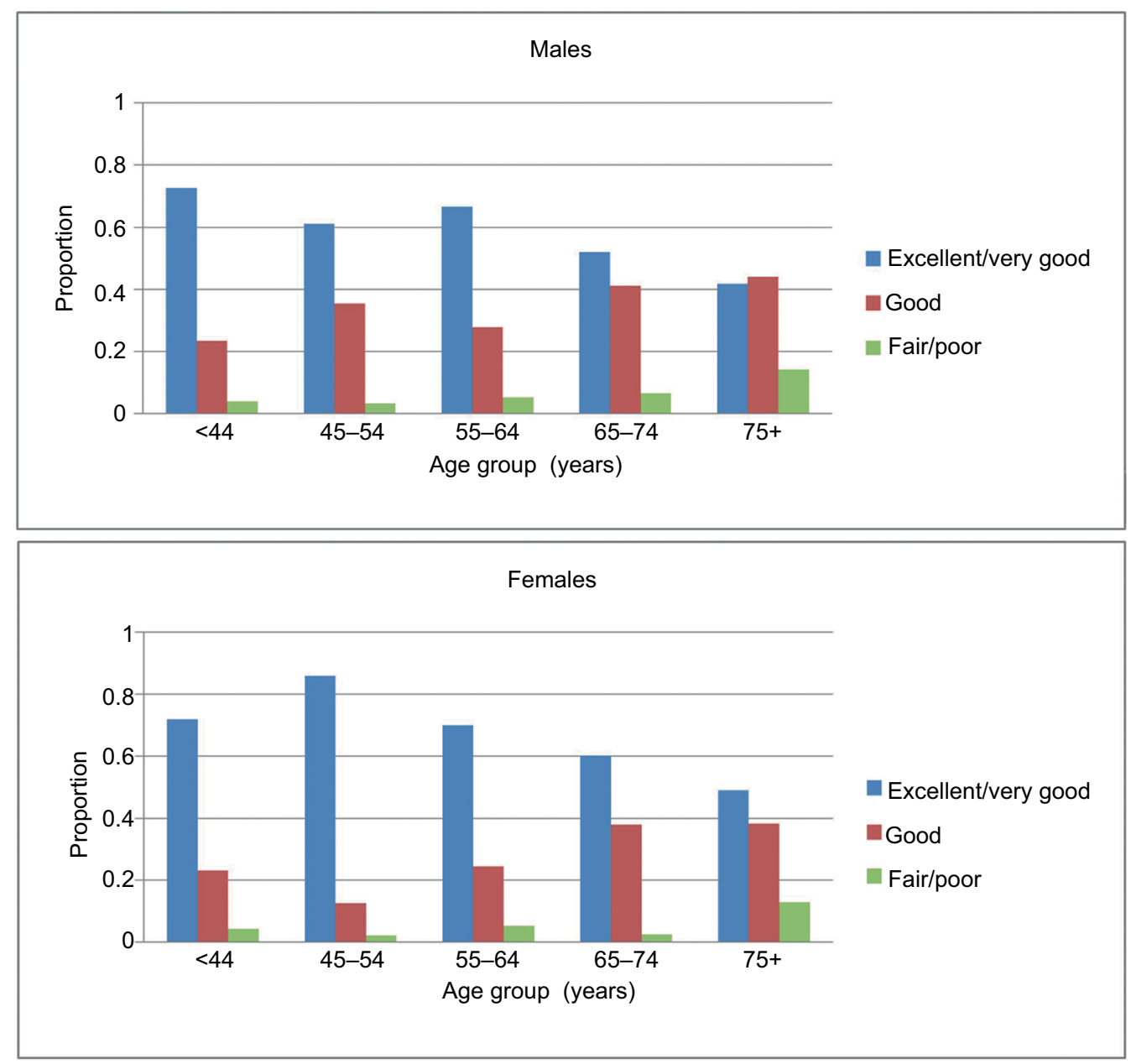

Figure I The relationship between age and self-rated health stratified by sex in the study cohort.

Note: Excellent self-rated health decreases with age in both males and females.

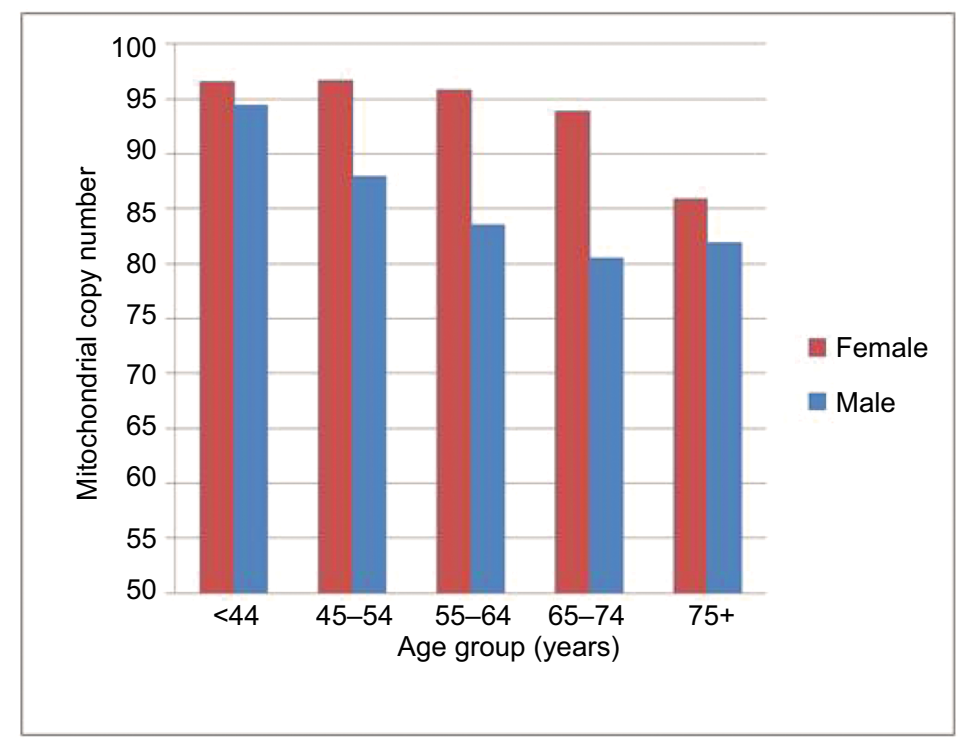

Figure 2 The relationship between age and mtDNA copy number in the study cohort, stratified by sex.

Notes: Age is associated with a lower mtDNA copy number. The mtDNA copy number remains higher in women than in men.

Abbreviation: mtDNA, mitochondrial DNA. 
Table 2 Association between mitochondrial DNA copy number and perceived general health

\begin{tabular}{|c|c|c|c|}
\hline & $\begin{array}{l}\text { Model I }{ }^{\mathrm{a}} \text { : } \\
\text { OR }^{\mathrm{b}}(95 \% \mathrm{Cl})\end{array}$ & $\begin{array}{l}\text { Model 2a: } \\
\text { OR }^{\mathrm{b}}(95 \% \mathrm{Cl})\end{array}$ & $\begin{array}{l}\text { Model 3a: } \\
\text { OR }^{\mathrm{b}}(95 \% \mathrm{Cl})\end{array}$ \\
\hline Age: linear & $0.32(0.10,0.99)$ & $11.70(3.09,43.36)$ & $|4.0|(3.44,55.70)$ \\
\hline quadratic & $0.12(0.06,0.24)$ & $2.52(1.01,6.32)$ & $\mathrm{I} .40(0.53,3.7 \mathrm{I})$ \\
\hline Female sex & $1.35(1.02,1.78)$ & $\mathrm{I} .44(1.07,1.94)$ & I.3I $(0.96,1.79)$ \\
\hline Number of comorbidities & - & $0.63(0.58,0.168)$ & $0.65(0.60,0.70)$ \\
\hline Body mass index: linear & - & - & $0.03(0.01,0.13)$ \\
\hline quadratic & & & $0.31(0.06,1.70)$ \\
\hline $\begin{array}{l}\text { Mitochondrial copy number, } \\
\text { log-transformed }\end{array}$ & $2.33(1.26,4.39)$ & $2.28(1.18,4.47)$ & $2.01(1.00,4.09)$ \\
\hline
\end{tabular}

Notes: Model I: adjusting for age (in a quadratic form) and sex; Model 2: adjusting for age (in a quadratic form), sex, and number of comorbidities; Model 3: adjusting for age (in a quadratic form), sex, number of comorbidities, and body mass index (in a quadratic form). ${ }^{\circ} \mathrm{OR}$ is the odds of having perceived general health in the better direction, compared to the odds in the worse direction for a given category.

The oxidative stress from free radicals may be responsible for the aging process which is tied to the mitochondria. ${ }^{29}$

The relationship between mtDNA copy number and mitochondrial function may vary. It may be that increased mtDNA copy number is not related to function. In rat models, a decline in mtDNA was not associated with a change in cytochrome $\mathrm{c}$ oxidase (COX) subunit I and III transcript levels in the liver and heart. ${ }^{31}$ In a normative sample of both cancer patients and a healthy cohort, quality of life decreased with age. Quality of life can also decrease with illness. ${ }^{32}$ There is clinical utility of self-rated health as an outcome. In previous work, poor selfrated health has been associated with adverse health outcomes such as hospitalization and emergency department visits. ${ }^{2}$ In cancer patients, quality of life at diagnosis is prognostic for mortality. ${ }^{33}$ Thus, the association of self-rated health with mtDNA copy number has potential clinical significance; however, additional research is needed to determine the direct link between mtDNA copy number and clinical outcomes.

This study has some limitations. As it is a cross-sectional study, one cannot draw causality from the findings. The potential for reporting bias is possible; however, no participants were aware of their genetic information while filling out the questionnaire. The largest limitation is the potential for an unknown confounder, such as medication use (some medications such as lithium are reported to improve mitochondrial function). There is likely some selection bias as patients within the biobank were more highly educated than the US national population and less likely to smoke. ${ }^{18}$ As further studies evaluate mtDNA copy number and potential exposures that lower those numbers, one can account for them in models.

\section{Conclusion}

In this study, we found an association of lower self-rated health with lower mtDNA copy number. This finding provides support to the concept that there may be a link between mtDNA copy number and how one feels. Given that mitochondrial function is influenced by several risk factors (e.g., socioeconomic status and environmental exposures) associated with health outcomes, it is possible that mtDNA copy number can serve as a biomarker that reflects collective effects of those risk factors, which in turn influences how one feels and health outcomes simultaneously. Future studies will need to evaluate mtDNA as a biomarker for clinical outcomes such as hospitalization or mortality to bring this idea closer to clinical practice.

\section{Acknowledgments}

The authors thank all the participants in the Mayo Clinic Biobank. The abstract of this paper was presented at the European Union Geriatric Society Meeting as a poster presentation with interim findings. The poster's abstract was published in European Geriatric Medicine (https://eugms2017-abstracts. elsevierdigitaledition.com/files/assets/common/downloads/ publication.pdf).

\section{Author contributions}

All authors contributed toward data analysis, drafting and revising the paper and agree to be accountable for all aspects of the work.

\section{Disclosure}

The authors report no conflicts of interest in this work.

\section{References}

1. Assari S. General self-efficacy and mortality in the USA: racial differences. J Racial Ethn Health Disparities. 2017;4(4):746-757.

2. Takahashi PY, Ryu E, Olson JE, et al. Health behaviors and quality of life predictors for risk of hospitalization in an electronic health recordlinked biobank. Int J Gen Med. 2015;8:247-254.

3. Barger SD, Cribbet MR, Muldoon MF. Participant-reported health status predicts cardiovascular and all-cause mortality independent of established and nontraditional biomarkers: evidence from a representative US sample. J Am Heart Assoc. 2016;5(9). pii: e003741. 
4. Jylha M, Volpato S, Guralnik JM. Self-rated health showed a graded association with frequently used biomarkers in a large population sample. J Clin Epidemiol. 2006;59(5):465-471.

5. Christian LM, Glaser R, Porter K, Malarkey WB, Beversdorf D, Kiecolt-Glaser JK. Poorer self-rated health is associated with elevated inflammatory markers among older adults. Psychoneuroendocrinology. 2011;36(10):1495-1504.

6. Dantzer R, Walker AK. Is there a role for glutamate-mediated excitotoxicity in inflammation-induced depression? J Neural Transm (Vienna). 2014;121(8):925-932.

7. Bratic A, Larsson NG. The role of mitochondria in aging. J Clin Invest. 2013;123(3):951-957.

8. Currais A. Ageing and inflammation - a central role for mitochondria in brain health and disease. Ageing Res Rev. 2015;21:30-42.

9. van Horssen J, van Schaik P, Witte M. Inflammation and mitochondrial dysfunction: a vicious circle in neurodegenerative disorders? Neurosci Lett. Epub 2017 Jun 28.

10. Lopez-Armada MJ, Riveiro-Naveira RR, Vaamonde-Garcia C, Valcarcel-Ares MN. Mitochondrial dysfunction and the inflammatory response. Mitochondrion. 2013;13(2):106-118.

11. Pawelec G, Goldeck D, Derhovanessian E. Inflammation, ageing and chronic disease. Curr Opin Immunol. 2014;29:23-28.

12. Tin A, Grams ME, Ashar FN, et al. Association between mitochondrial DNA copy number in peripheral blood and incident CKD in the atherosclerosis risk in communities study. J Am Soc Nephrol. 2016;27(8):2467-2473.

13. Masayesva BG, Mambo E, Taylor RJ, et al. Mitochondrial DNA content increase in response to cigarette smoking. Cancer epidemiology, biomarkers \& prevention: a publication of the American Association for Cancer Research, cosponsored by the American Society of Preventive Oncology. 2006;15(1):19-24.

14. Mengel-From J, Thinggaard M, Dalgard C, Kyvik KO, Christensen K, Christiansen L. Mitochondrial DNA copy number in peripheral blood cells declines with age and is associated with general health among elderly. Hum Genet. 2014;133(9):1149-1159.

15. Knez J, Winckelmans E, Plusquin M, et al. Correlates of peripheral blood mitochondrial DNA content in a general population. Am J Epidemiol. 2016;183(2):138-146.

16. Nicolson GL. Mitochondrial dysfunction and chronic disease: treatment with natural supplements. Integr Med (Encinitas). 2014;13(4): 35-43.

17. Olson JE, Rohrer Vitek CR, Bell EJ, et al. Participant-perceived understanding and perspectives on pharmacogenomics: the Mayo Clinic RIGHT protocol (right drug, right dose, right time). Genet Med 2017;19(7):819-825.

18. Olson JE, Ryu E, Johnson KJ, et al. The Mayo Clinic Biobank: a building block for individualized medicine. Mayo Clin Proc. 2013;88(9):952-962.
19. Jun G, Flickinger M, Hetrick KN, et al. Detecting and estimating contamination of human DNA samples in sequencing and array-based genotype data. Am J Hum Genet. 2012;91(5):839-848.

20. Manichaikul A, Mychaleckyj JC, Rich SS, Daly K, Sale M, Chen WM. Robust relationship inference in genome-wide association studies. Bioinformatics. 2010;26(22):2867-2873.

21. Zheng X, Levine D, Shen J, Gogarten SM, Laurie C, Weir BS. A highperformance computing toolset for relatedness and principal component analysis of SNP data. Bioinformatics. 2012;28(24):3326-3328.

22. Andrews RM, Kubacka I, Chinnery PF, Lightowlers RN, Turnbull DM, Howell N. Reanalysis and revision of the Cambridge reference sequence for human mitochondrial DNA. Nat Genet. 1999;23(2):147.

23. Ding J, Sidore C, Butler TJ, et al. Assessing mitochondrial DNA variation and copy number in lymphocytes of $\sim 2,000$ Sardinians using tailored sequencing analysis tools. PLoS Genet. 2015;11(7):e1005306.

24. Weissensteiner H, Pacher D, Kloss-Brandstatter A, et al. HaploGrep 2: mitochondrial haplogroup classification in the era of high-throughput sequencing. Nucleic Acids Res. 2016;44(W1):W58-63.

25. St Sauver JL, Boyd CM, Grossardt BR, et al. Risk of developing multimorbidity across all ages in an historical cohort study: differences by sex and ethnicity. BMJ Open. 2015;5(2):e006413.

26. Wachsmuth M, Hubner A, Li M, Madea B, Stoneking M. Age-related and heteroplasmy-related variation in human mtDNA copy number. PLoS Genet. 2016;12(3):e1005939.

27. Hebert SL, Marquet-de Rouge P, Lanza IR, et al. Mitochondrial aging and physical decline: insights from three generations of women. $J$ Gerontol A Biol Sci Med Sci. 2015;70(11):1409-1417.

28. Zole E, Zadinane K, Pliss L, Ranka R. Linkage between mitochondrial genome alterations, telomere length and aging population. Mitochondrial DNA A DNA Mapp Seq Anal. 2017:1-8.

29. Lane N. A unifying view of ageing and disease: the double-agent theory. J Theor Biol. 2003;225(4):531-540.

30. Short KR, Bigelow ML, Kahl J, et al. Decline in skeletal muscle mitochondrial function with aging in humans. Proc Natl Acad Sci USA. 2005;102(15):5618-5623.

31. Barazzoni R, Short KR, Nair KS. Effects of aging on mitochondrial DNA copy number and cytochrome c oxidase gene expression in rat skeletal muscle, liver, and heart. J Biol Chem. 2000;275(5):3343-3347.

32. Singh JA, Satele D, Pattabasavaiah S, Buckner JC, Sloan JA. Normative data and clinically significant effect sizes for single-item numerical linear analogue self-assessment (LASA) scales. Health Qual Life Outcomes. 2014;12:187.

33. Sloan JA, Zhao X, Novotny PJ, et al. Relationship between deficits in overall quality of life and non-small-cell lung cancer survival. J Clin Oncol. 2012;30(13):1498-1504.

34. Jun G, Flickinger M, Hetrick KN, et al. Detecting and estimating contamination of human DNA samples in sequencing and array-based genotype data. Am J Hum Genet. 2012;91(5):839-848.
The Application of Clinical Genetics

\section{Publish your work in this journal}

The Application of Clinical Genetics is an international, peer-reviewed open access journal that welcomes laboratory and clinical findings in the field of human genetics. Specific topics include: Population genetics; Functional genetics; Natural history of genetic disease; Management of genetic disease; Mechanisms of genetic disease; Counselling and ethical
Dovepress

issues; Animal models; Pharmacogenetics; Prenatal diagnosis; Dysmorphology. The manuscript management system is completely online and includes a very quick and fair peer-review system, which is all easy to use. Visit http://www.dovepress.com/testimonials.php to read real quotes from published authors. 